\title{
Lack of association between Hashimoto thyroiditis and breast cancer: A quantitative research synthesis
}

\author{
Nicholas J. Sarlis ${ }^{1}$, Loukas Gourgiotis ${ }^{1}$, Frank Pucino ${ }^{2}$, George J. Tolis ${ }^{3}$ \\ ${ }^{1}$ Clinical Endocrinology Branch, National Institute of Diabetes and Digestive and Kidney Diseases (NIDDK), Nation- \\ al Institutes of Health (NIH), Bethesda, MD, USA, ${ }^{2}$ Dept. of Pharmacy, Clinical Center, NIH, Bethesda, MD, USA, \\ and ${ }^{3}$ Division of Endocrinology, Dept. of Medicine, Hippocrateion Athens General Hospital, Athens, Greece.
}

\begin{abstract}
Several authors have suggested a positive association between Hashimoto thyroiditis (HT) and breast cancer (BrCa). Others have refuted these findings; hence, this subject remains controversial. We therefore reviewed the world literature on this subject accumulated over the last 50 years and performed a quantitative research synthesis, a meta-analysis variant. The incidence risk ratios and $95 \%$ confidence intervals (CI's) were calculated for each study and the combined relative risk (RR) was estimated. We found 37 relevant studies, of which only 13 were accessible to analysis. A significant association $(R R=1.40 ; C I=1.29-1.53, P<0.022)$ was found for 6 of the 13 studies pertaining to 1,431 women. However, in the cumulative population of 14,226 women (from all 13 studies), we failed to demonstrate an association between the diagnoses of HT and $B r C a(R R=1.07$; $\mathrm{CI}=0.99-1.15 ; \mathbf{P}=\mathbf{0 . 0 8}$ ). In conclusion, we believe that selection bias or institutional referral bias, in at least some of the "positive" studies, may have led to the spurious recognition of an association between HT and BrCa, especially as both of these conditions are highly prevalent in women between the 4th and 7 th decade of life.
\end{abstract}

Key-words: antithyroidal antibodies, breast cancer, Hashimoto thyroiditis, meta-analysis, thyroid gland

\section{INTRODUCTION}

Breast cancer $(\mathrm{BrCa})$ is the most common cancer in women in the Western world, but despite intensive research only a part of its incidence is believed to be attributable to known risk factors, either genetic or epige-

Address Correspondence to:

Nicholas J. Sarlis, MD, PhD, CEB/NIDDK/NIH, Bldg. 10; Rm. 8D12C, 10 Center Drive - MSC 1758, Bethesda, MD 20892-1758 U.S.A., Phone: 301-435-9267, FAX: 301-402-4136,

E-mail: NJSarlis@helix.nih.gov

Received 07-09-2001, Revised 30-10-2001, Accepted 12-12-2001 netic $^{1}$. Several common medical conditions are associated with changes in the endocrine milieu, which secondarily may influence the risk of development and progression of $\mathrm{BrCa}^{2}$. One of these conditions, which has been positively linked by some authors to $\mathrm{BrCa}$, is Hashimoto thyroiditis (HT). The mechanism for this association is not clear, although some studies have shown increased prevalence of antithyroidal auto-antibodies in women with $\mathrm{BrCa}$, compared to healthy controls, thus leading to the hypothesis that the mammary tumor and the thyroid gland may share common antigens, such as thyroid peroxidase (TPO) and sodium-iodide ( $\mathrm{Na}+/ \mathrm{I}-)$ symport- 
$\mathrm{er}^{3}$. However, the above findings have been refuted by other authors ${ }^{4,5}$. Hence, the subject remains controversial.

We therefore reviewed the relevant world literature spanning the last 50 years, and performed a quantitative research synthesis, a variant of meta-analysis. Despite a strong association suggested by some of the studies, these involved only a small proportion of the total number of cases cumulatively reported. When considering the total population of women in the analysis of the statistically evaluable studies, no significant association became evident between these two disorders.

\section{DATA SOURCE, OPERATIONAL DEFINITIONS AND METHODS}

\section{Biomedical literature}

MEDLINE and manual literature searches (19502001) were conducted using the Medical Subject Headings (MESH) "Hashimoto", "thyroiditis", "hypothyroidism", "thyroid disease", "anti-thyroidal antibodies", and "goiter" in conjunction with the MESH terms "breast cancer" and "breast neoplasms". Both English and nonEnglish-language articles were reviewed. We selected case series, cross-sectional studies (or prevalence surveys), "case-control" (retrospective) studies, as well as retrospective cohort/clinical cohort studies examining: either (i) the prevalence of thyroid disease (or in some cases, HT specifically) in women with $\mathrm{BrCa}$ (as well as "healthy controls" - whenever applicable) or (ii) the prevalence of $\mathrm{BrCa}$ in women with HT (as well as "healthy controls" - whenever applicable). Notably, in some of the studies focusing on the prevalence of thyroid disease in women with $\mathrm{BrCa}$ versus "control" patients, the latter population also included women with benign breast disease.

\begin{tabular}{ll}
\hline $\begin{array}{ll}\text { Abbreviations } \\
\text { Antibodies }\end{array}$ & \\
Breast cancer & $\mathrm{Ab}$ 's \\
Free thyroxine & $\mathrm{BrCa}$ \\
Hashimoto thyroiditis & $\mathrm{fT}$ \\
Not available & $\mathrm{N} / \mathrm{A}$ \\
Non-significantly different & $\mathrm{NSD}$ \\
Patients & Pts \\
Thyroidal \% radioiodine uptake $(24 \mathrm{H})$ & \% RAIU \\
Relative risk & RR \\
Tri-iodothyronine & T3 \\
Thyroxine & T4 \\
Thyroid peroxidase & TPO \\
Thyrotropin & TSH \\
Ultrasound & $\mathrm{U} / \mathrm{S}$
\end{tabular}

\section{Operational Definitions and Systematic Evaluation of Studies}

In view of the variety of parameters studied and the conflicting results among published reports (due, in part, to the heterogeneity of the corresponding patient populations), we selected only those studies that were amenable to statistical analysis. The criteria for selection of studies were defined a priori. For the purposes of our analysis, we defined HT as the presence of: (i) positive anti-thyroidal antibodies (ATA's), i.e. high titers of antimicrosomal or antithyroglobulin (anti-Tg) antibodies (measured as inverse fractions of dilution in hemagglutination assays in earlier studies) or high levels of antithyroid peroxidase (anti-TPO) or anti-Tg antibodies levels (measured as $\mathrm{mU} / \mathrm{l}$ in specific immunoassays in more recent studies), or (ii) elevated serum thyrotropin (TSH) levels or (iii) decreased serum total triiodothyronine (T3) levels or (iv) the presence of nodular goiter by physical exam and/or ultrasonographic evaluation. We took into consideration the fact that the cardinal cause for conditions (ii)-(iv) in the study populations would be HT (6). Additionally, data were extracted for further analysis from studies meeting all of the following criteria: (i) a "healthy control" group of patients was included, (ii) clinical characteristics of various patient groups were reported, and (iii) the diagnosis of $\mathrm{BrCa}$ was based on either histopathologic confirmation of the malignancy or patient record review. Each study was independently reviewed by two of the authors (L.G. and N.J.S.) and, subsequently, the methodology and results thereof were summarized into tables prior to formal statistical analysis.

\section{Statistical analysis}

The method of quantitative research synthesis, a meta-analysis variant, was used to analyze the available data $^{7,8}$. This method features the following components: (i) incidence risk ratio calculation: for each individual study, a treatment effect size (relative incidence risk ratio) and 95\% confidence interval (CI) were calculated by commercially available statistical software (SAS/ STAT ${ }^{\circledR}$, Cary, NC) ${ }^{9}$; (ii) common relative risk (RR) calculation: the Mantel-Haenszel test was used for that purpose; (iii) common treatment effect estimation. Additionally, the Breslow-Day test of homogeneity $\left(\chi^{2}\right.$-test statistic) was used to assess whether any inter-study differences would be attributable to random sampling alone (depending on the distribution of effect sizes among studies). If all studies are homogeneous (e.g., the presence of HT consistently increased the risk of detection/diagnosis of $\mathrm{BrCa}$ ), this $\chi^{2}$-statistic would be non-significant. Conversely, with this method, if the heterogeneity (in- 
Table 1. Characteristics of published studies $(n=29)$

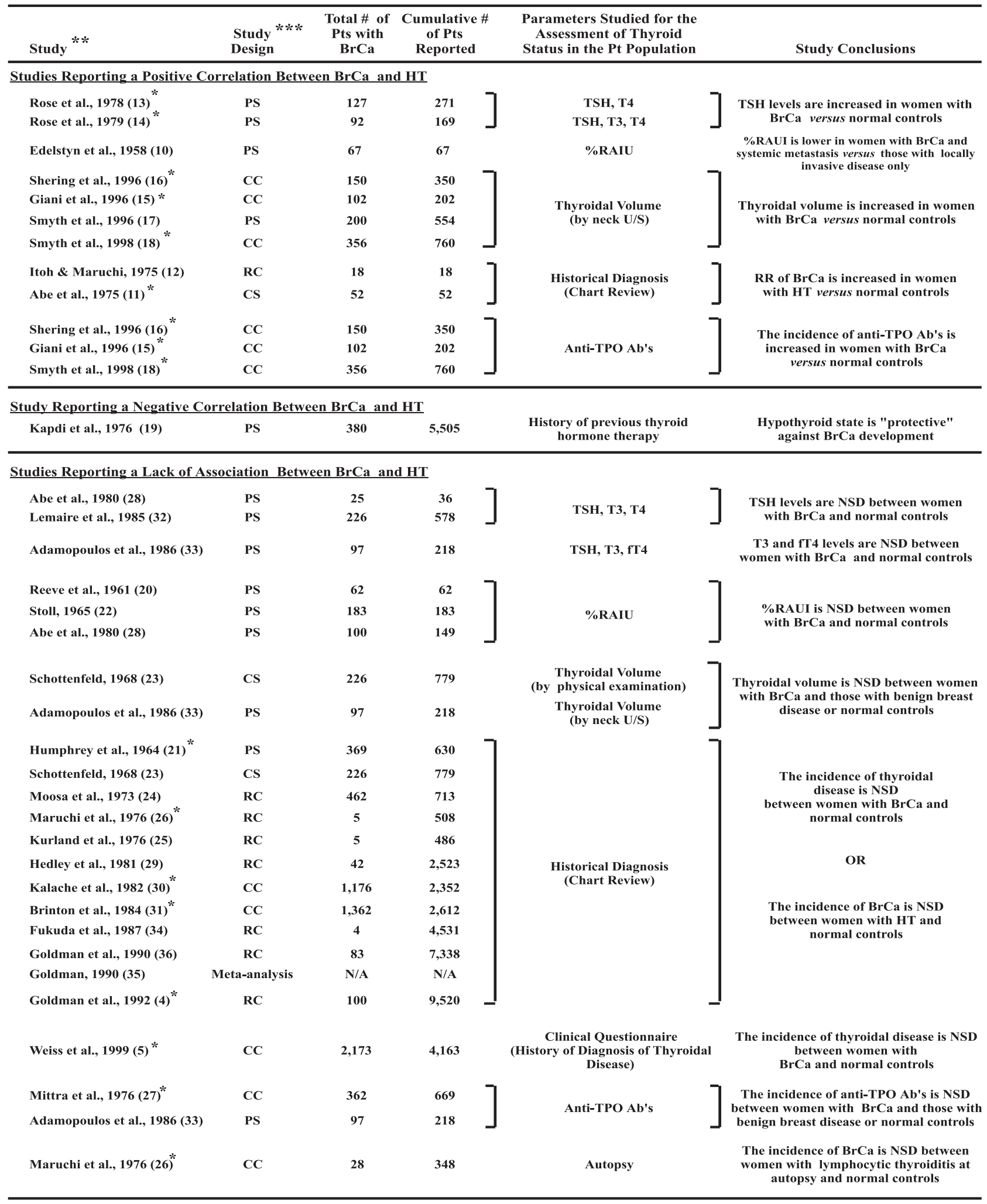

* : Studies evaluated in the quantitative research synthesis/meta-analysis

** : CC $=$ Case-Control; CS $=$ Case Series; PS: Prevalence Survey (Cross-Sectional Study); RC=Retrospective Cohort

$* * *$ : Some of the studies appear more than once in this table, as they report on several different parameters of thyroid status assessment 
ter-study variability) was large, then the Breslow-Day $\chi^{2}$ statistic was significant. Further, in this analysis, CI's are typically wider than those derived by a simpler statistical method, such as the inverse-variance weighted average comparison ${ }^{7}$. For all statistical tests, a two-tailed $\mathrm{P}$ value of $<0.05$ was considered significant.

\section{RESULTS}

From our literature search, we found 34 relevant reports on the subject, 29 of which $(85 \%)$ are summarized in table $1^{4,5,10-36}$. The remaining 5 reports $(15 \%)$ were editorials, comments, letters and opinion statement type of publications and, hence, were not amenable to quantitative analysis ${ }^{37-41}$. Taking into account the selection criteria mentioned in the Methods section above, only 13 of the 29 studies $(45 \%)$ cited in table 1 were evaluable (designated by an asterisk [ $\left.{ }^{*}\right]$ in Table 1$)^{4,5,11,13-16,18,21,26,27,29,31}$. The primary reason for excluding $>50 \%$ of the published studies was the rather poor quality of the reported data, which led to their failure to meet the defined selection criteria for our analysis. Six of the 13 statistically evaluable studies $(46 \%)$ showed a significant association between $\mathrm{HT}$ and $\mathrm{BrCa}(\mathrm{RR}=1.40, \mathrm{CI}=1.29-1.53, \mathrm{P}<0.022$, by Fisher's exact test $)^{11,13-16,18}$. These results were perti- nent for a cumulative population of 1,431 women (Figure 1).

However, in the cumulative number of 14,226 women (from all 13 evaluable studies), we failed to demonstrate an association between the diagnoses of HT and $\mathrm{BrCa}(\mathrm{RR}=1.07$; $\mathrm{CI}=0.99-1.15, \mathrm{P}=0.08$, by Fisher's exact test), as is clearly evident in figure 1 .

Effect sizes for these studies were not homogeneous $(p=0.001)$, as assessed by the Breslow-Day test of homogeneity. As noted above, in this test statistical significance is reached when the population size among studies is heterogeneous ${ }^{7}$. However, homogeneity across studies $(p>0.05)$ was observed after removing 3 studies which, in fact, were those "positive" studies reporting the most significant incidence risk ratio values ${ }^{14-16}$.

\section{DISCUSSION}

The association between $\mathrm{HT}$ and $\mathrm{BrCa}$ has been suggested by several epidemiological and clinical cohort studies ever since Sir George Beatson, surgeon at the Glasgow Cancer Hospital, experimented with thyroid hormones as a treatment for BrCa in $1896^{42}$. Sixty years later, Loeser observed that hyperthyroidism protected

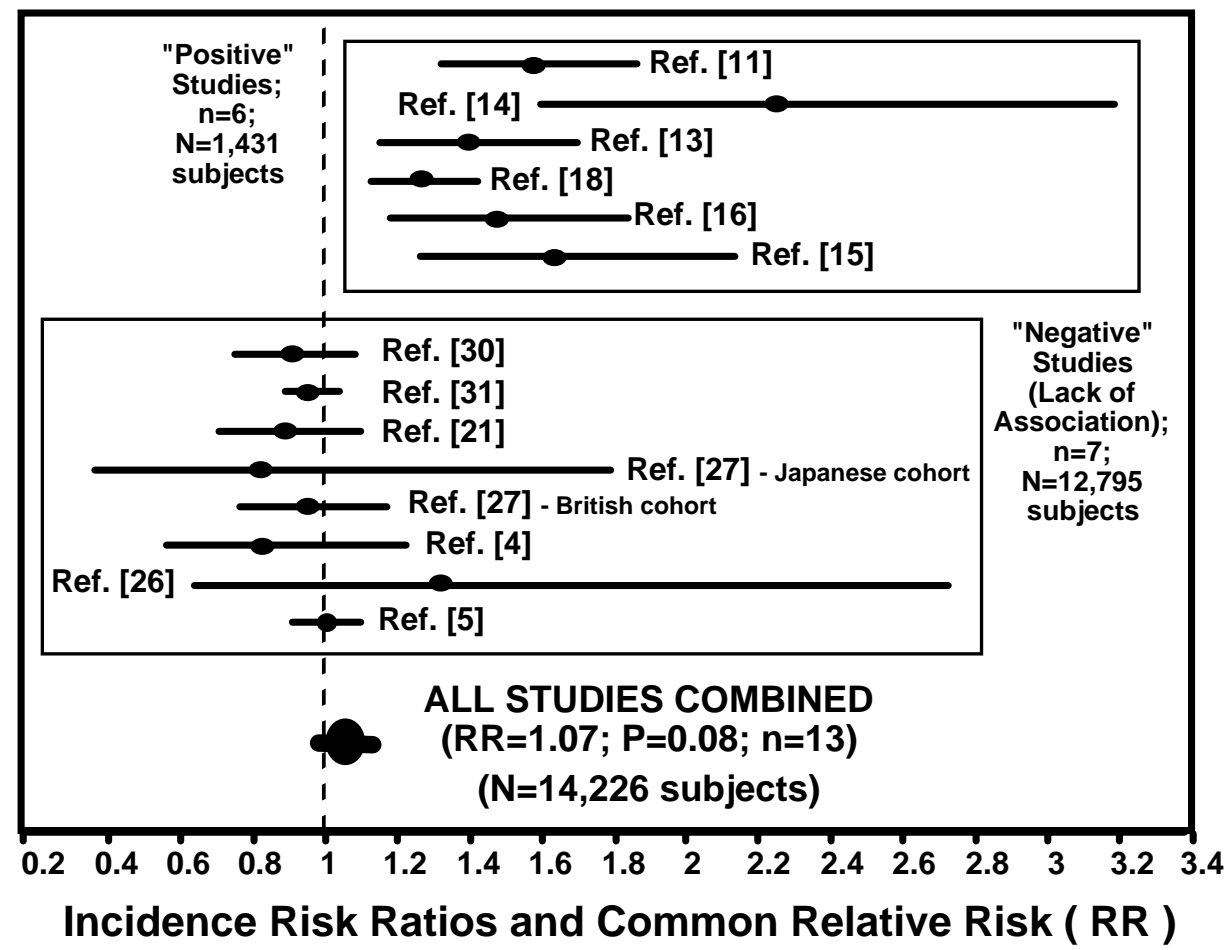

Figure 1. Incidence risk ratios for each individual study and derived common relative risk (RR) for BrCa in women with HT in all studies $(\mathrm{n}=13)$. Corresponding confidence intervals for all values are shown as horizontal bars. 
against $\mathrm{BrCa}$, whereas women who had undergone subtotal thyroidectomy developed $\mathrm{BrCa}$ more often than expected and, thus, recommended administration of thyroid hormone as a treatment for $\mathrm{BrCa}^{43}$. This author also postulated that the lack of thyroid hormones permitted the development of malignant cells within the mammary epithelium ${ }^{43}$.

Since that time, several reports have suggested an association between thyroid diseases, or their treatments, and the occurrence of $\mathrm{BrCa}^{11,13-16,18}$. However, both HT and $\mathrm{BrCa}$ are highly prevalent in women between the 4th and 7th decade of life and, hence, there may be adequate reason to attribute the purported association to chance alone ${ }^{1,6,44-46}$. To further clarify the issue, we performed a systematic review of the relevant literature over the last 50 years, which had yielded conflicting results among the various reports.

The strongest basis for a positive association between $\mathrm{HT}$ and $\mathrm{BrCa}$ has been the observation that women with this malignancy have higher prevalence of anti-TPO antibodies, as well as higher mean thyroidal volumes versus healthy controls ${ }^{17,18}$. However, increased prevalence of ATA's has also been observed in women with benign breast disease ${ }^{33}$. Further, the pathophysiologic basis of any association between the presence of ATA's, goiter, or hypothyroidism and mammary epithelial proliferation/ tumor formation is very tenuous. Moreover, the "positive" studies (those reporting an association between HT and $\mathrm{BrCa}$ ) pertain to women from widely divergent ethnic backgrounds (i.e. Western European, US-Caucasian, Japanese) with different iodine sufficiency status, as well as genetic and environmental risk factor profiles. This fact needs to be taken into consideration when raising the possibility of a true biologically pertinent association between these two diseases. Finally, even when the 6 "positive" studies are combined, the cumulative number of subjects therein, i.e. 1,431 women, remains much lower than that in all 13 studies, both "positive" and "negative", i.e. 14,226 women, reflecting the rather small size of individual "positive" studies.

In contrast to the above, 7 additional studies have failed to verify the alleged association between HT and $\mathrm{BrCa}^{4,5,21,26,27,29,31}$. Indeed, when data from these studies (showing lack of association between the two disorders) are pooled together with the 6 "positive" ones, no statistically significant association is found. Notably, the combined population sample for our analysis includes 14,226 women and, thus, achieves considerable statistical power for this observation.
The investigation of a possible relationship between $\mathrm{HT}$ and, indeed, thyroid disease in general, and $\mathrm{BrCa}$ has been pursued vigorously in the literature over many decades ${ }^{2-5,10-43}$. The lack of consistency in the findings of the studies performed heretofore, as well as the possibility of selection bias or institutional referral bias ${ }^{47}$ in at least some of the "positive" studies, suggest that $\mathrm{HT}$ and $\mathrm{BrCa}$ are most probably not causally related, but may share common etiologic factors which remain unidentified.

With regard to the possible etiology of any relationship between thyroid disease and $\mathrm{BrCa}$, a novel insight has recently been provided by studies examining the effect of cytokines, and particularly interleukin (IL)-6, on thyroid function. Serum levels of tumor necrosis factor (TNF) and IL-6 have been shown to be elevated in patients with non-thyroidal illness, including those with assorted malignancies, and to be associated with reduced mean serum total and free T3 levels, as well as occasionally low serum total and free thyroxine (T4) levels ${ }^{40,41,48-51}$. Further, thyroid dysfunction can occasionally be observed in cancer patients receiving IL-2-based immunothera$\mathrm{py}^{52,53}$. Specifically for $\mathrm{BrCa}$, in a study of women with recurrence of this malignancy who did not respond to chemotherapy, serum levels of IL- 6 and IL- 8 were significantly higher than those seen in women who showed a partial response ${ }^{41}$. Moreover, in this study, a strong negative correlation was observed between serum IL-6 and free T3 (as well as free T4) levels in the non-respond$\mathrm{ers}^{41}$. Thus, it is plausible that circulating (or intrathyroidally produced) IL-6 may lead to a hypothyroid state in at least some of the patients with recurrent metastatic $\mathrm{BrCa}$. Further research in this area, elucidating the relationship between thyroid function and serum levels of IL-6 (or other pro-inflammatory cytokines) is certainly warranted and could potentially explain the wide discrepancies among serum levels of thyroid hormones reported in the published studies.

Limitations of our analysis include the following: (i) the studies taken into consideration reported measurements and assessments of diverse parameters, which were generally not directly comparable; (ii) we cannot rule out the possibility that some of the patients with simple goiter and/or primary hypothyroidism did not have HT as their underlying diagnosis; (iii) more than $50 \%$ of the published studies on the subject were excluded due to the rather poor quality of the reported data thus rendering them inaccessible to statistical evaluation; and (iv) in any meta-analysis spanning an extended period of time (50 years in our case), there is a possibility that not all of the relevant publications were traced. In this connection, 
data suggestive of lack of association between any two diseases may never be reported due to publication bias; hence, the number of "negative" studies in our analysis may be underestimated.

In conclusion, in a research synthesis, a meta-analysis variant, of 13 studies involving a combined cohort of 14,226 women, we failed to demonstrate a statistically significant association between the diagnoses of HT and $\mathrm{BrCa}$. It is likely that previous reports suggesting a positive correlation between these two entities may have been flawed by analysis design, strong institutional bias, and selection of patient populations with very advanced $\mathrm{BrCa}$ exhibiting features consistent with non-thyroidal illness, such as intense hypercytokinemia.

\section{ACKNOWLEDGMENTS}

We would like to thank Drs. Jacob Robbins and Paul M. Yen, both at the National Institute of Diabetes and Digestive and Kidney Diseases (NIDDK), NIH, Bethesda, MD, for a thorough review of this manuscript and their insightful recommendations. We also appreciate the constructive comments by Dr. Seth M. Steinberg, of the National Cancer Institute, NIH, which increased the statistical robustness of our analysis and optimized the presentation of the data. Finally, we thank Dr. Shinishiro Ando, of NIDDK, NIH, for providing the translation of papers published in the Japanese literature.

\section{REFERENCES}

1. Couch FJ, Weber BL, 1998 Breast cancer. In: Vogelstein B, Kinzler KW (eds). New York, NY: McGraw-Hill pp 537-563.

2. Smyth PP, 1997 The thyroid and breast cancer: a significant association? Ann Med 29: 189-191.

3. Smyth PP, 2000 Autoimmune thyroid disease and breast cancer: a chance association? J Endocrinol Invest 23: 4243.

4. Goldman MB, Monson RR, Maloof F, 1992 Benign thyroid diseases and the risk of death from breast cancer. Oncology 49: 461-466.

5. Weiss HA, Brinton LA, Potischman NA, Brogan D, Coates RJ, Gammon MD, Malone KE, Schoenberg JB 1999 Breast cancer risk in young women and history of selected medical conditions. Int J Epidemiol. 28: 816-823.

6. Dayan CM, Daniels GH, 1996 Chronic autoimmune thyroiditis. N Engl J Med 335: 99-107.

7. Fletcher RH, Fletcher SW, Wagner EH, 1996 Clinical Epidemiology. The Essentials (3rd ed) Baltimore, MD: Williams and Wilkins: 235-366.

8. Csako G, Byrd D, Wesley RA, Sarlis NJ, Skarulis MC, Nieman LK, Pucino F, 2000 Assessing the effects of thy- roid suppression on benign solitary thyroid nodules. A model for using quantitative research synthesis. Medicine (Baltimore) 79: 9-26.

9. Anonymous, 1990 SAS/STAT ${ }^{\circledR}$ User guide: (Version 6.4th Ed.) Cary, NC, USA: SAS Institute.

10. Edelstyn GA, Lyons AR, Welbourn RB. 1958 Thyroid function in patients with mammary cancer. Lancet i.: 670671.

11. Abe R, Taneichi Y, Kumagaya N, Terui K 1975 Thyroid gland function on breast neoplasms. Horumon To Rinsho 23: 133-136 [In Japanese].

12. Itoh K, Maruchi N, 1975 Breast Cancer in patients with Hashimoto's thyroiditis. Lancet ii: 1119-1121.

13. Rose DP, Davis TE, 1978 Plasma thyroid-stimulating hormone and thyroxine concentrations in breast cancer. Cancer 41: 666-669.

14. Rose DP, Davis TE, 1979 Plasma triiodothyronine concentrations in breast cancer. Cancer 43: 1434-1438.

15. Giani C, Fierabracci P, Bonacci R, Gigliotti A, Campani D, De Negri F, Cecchetti D, Martino E, Pinchera A, 1996 Relationship between breast cancer and thyroid disease: relevance of autoimmune thyroid disorders in breast malignancy. J Clin Endocrinol Metab 81: 990-994.

16. Shering SG, Zbar AP, Moriarty M, McDermott EW, O'Higgins NJ, Smyth PP, 1996 Thyroid disorders and breast cancer. Eur J Cancer Prev 5: 504-506.

17. Smyth PP, Smith DF, McDermott EW, Murray MJ, Geraghty JG, O'Higgins NJ, 1996 A direct relationship between thyroid enlargement and breast cancer. J Clin Endocrinol Metab 81: 937-941.

18. Smyth PP, Shering SG, Kilbane MT, Murray MJ, McDermott EW, Smith DF, O'Higgins NJ, 1998 Serum thyroid peroxidase autoantibodies, thyroid volume, and outcome in breast carcinoma. J Clin Endocrinol Metab 83: 27112716.

19. Kapdi CC, Wolfe JN, 1976 Breast cancer. Relationship to thyroid supplements for hypothyroidism. JAMA 236: 1124-1127.

20. Reeve TS, Hales IB, Rundle FF, Myhill J, 1961 Thyroid function on the presence of Breast Cancer. Lancet i: 632634.

21. Humphrey LJ, Swerdlow M, 1964 The relationship of breast disease to thyroid disease. Cancer 17: 1170-1173.

22. Stoll B 1965 Breast cancer and hypothyroidism. Cancer 18: 1431-1436.

23. Schottenfeld D, 1968 The relationship of breast cancer to thyroid disease. J Chronic Dis 21: 303-313.

24. Moossa AR, Evans DA, Brewer AC, 1973 Thyroid status and breast cancer. Reappraisal of an old relationship. Ann R Coll Surg Engl 53: 178-188.

25. Kurland LT, Annegers JF, 1976 Breast cancer and Hashimoto thyroiditis. Lancet i: 808 [Letter].

26. Maruchi N, Annegers JF, Kurland LT, 1976 Hashimoto's thyroiditis and breast cancer. Mayo Clin Proc 51: 263-265.

27. Mittra I, Perrin J, Kumaoka S, 1976 Thyroid and other autoantibodies in British and Japanese women: an epidemiological study of breast cancer. Br Med J i: 257-259.

28. Abe R, Hirosaki A, Kimura M, 1980 Pituitary-thyroid 
function in patients with breast cancer. Tohoku J Exp Med 132: 231-236.

29. Hedley AJ, Jones SJ, Spiegelhalter DJ, Clements P, Bewsher PD, Simpson JG, Weir RD, 1981 Breast cancer in thyroid disease: fact or fallacy? Lancet i: 131-133.

30. Kalache A, Vessey MP, McPherson K, 1982 Thyroid disease and breast cancer: findings in a large case-control study. Br J Surg 69: 434-435.

31. Brinton LA, Hoffman DA, Hoover R, Fraumeni JF Jr, 1984 Relationship of thyroid disease and use of thyroid supplements to breast cancer risk. J Chronic Dis 37: 877893.

32. Lemaire M, Baugnet-Mahieu L, 1986 Thyroid function in women with breast cancer. Eur J Cancer Clin Oncol 22: 301-307.

33. Adamopoulos DA, Vassilaros S, Kapolla N, Papadiamantis J, Georgiakodis F, Michalakis A, 1986 Thyroid disease in patients with benign and malignant mastopathy. Cancer 57: $125-128$.

34. Fukuda A, Hirohata T, Noguchi S, Ikeda M, Matsuo K, Yoshida A, 1987 Risks for malignancies in patients with chronic thyroiditis: a long-term follow-up study. Jpn J Cancer Res 78: 1329-1334.

35. Goldman MB, 1990 Thyroid diseases and breast cancer. Epidemiol Rev 12: 16-28.

36. Goldman MB, Monson RR, Maloof F, 1990 Cancer mortality in women with thyroid disease. Cancer Res 50: 22832289.

37. Backwinkel K, Jackson AS, 1964 Some features of breast cancer and thyroid deficiency. Cancer 17: 1174-1176.

38. Saito S, Abe R, Sakurada T, Yoshida K, Kimura M, 1977 The thyroid reserve in patients with breast cancer. Tohoku J Exp Med 122: 229-23

39. Aldinger KA, Schultz PN, Blumenschein GR, Samaan NA, 1978 Thyroid stimulating hormone and prolactin levels in breast cancer. Arch Intern Med 138: 1638-1641.

40. Yokoe T, Iino Y, Takei H, Horiguchi J, Koibuchi Y, Mae- mura M, Ohwada S, Morishita Y, 1996 Relationship between thyroid-pituitary function and response to therapy in patients with recurrent breast cancer. Anticancer Res 16: 2069-2072.

41. Yokoe T, Iino Y, Takei H, Horiguchi J, Koibuchi Y, Maemura M, Ohwada S, Morishita Y, 1997 Changes of cytokines and thyroid function in patients with recurrent breast cancer. Anticancer Res 17: 695-699.

42. Beatson GT, 1896 On the treatment of inoperable cases of carcinoma of the mamma. Suggestions for a new method of treatment with illustrative cases. Lancet ii: 104-107 and 162-165.

43. Loeser AA, 1954 A new therapy for prevention of postoperative recurrences in genital and breast cancer. BMJ ii: $1380-1383$.

44. Phillips K-A, Glendon G, Knight JA, 1999 Putting the risk of breast cancer in perspective. N Engl J Med 340: 141-144.

45. Bjoro T, Holmen J, Kruger O, Midthjell K, Hunstad K, Schreiner T, Sandnes L, Brochman H, 2000 Prevalence of thyroid disease, thyroid dysfunction and thyroid peroxidase antibodies in a large unselected population. The Health Study of Nord Trondelag (HUNT). Eur J Endocrinol 143: 639-647.

46. Canaris GJ, Manowitz NR, Mayor G, Ridgway EC, 2000 The Colorado thyroid disease prevalence study. Arch Intern Med 160: 526-534.

47. Greenland S, Schwartzbaum JA, Finkle WD, 2000 Problems due to small samples and sparse data in conditional logistic regression analysis. Am J Epidemiol 151: 531-539.

48. Mooradian AD, Reed RL, Osterweil D, Schiffman R, Scuderi P, 1990 Decreased serum triiodothyronine is associated with increased concentrations of tumor necrosis factor. J Clin Endocrinol Metab 71: 1239-1242.

49. Boelen A, Platvoet-Ter Schiphorst MC, Wiersinga WM, 1993 Association between serum interleukin-6 and serum 3,5,3'-triiodothyronine in nonthyroidal illness. J Clin En- 